\title{
ICD-11 complex post-traumatic stress disorder: simplifying diagnosis in trauma populations
}

\author{
Marylène Cloitre
}

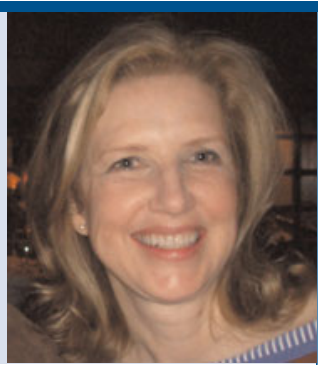

\section{Summary}

ICD-11 complex post-traumatic stress disorder (PTSD) is a new disorder that describes the more complex reactions that are typical of individuals exposed to chronic trauma. The addition of this disorder as distinct from PTSD is expected to provide greater precision in the diagnosis of trauma populations and more personalised and effective treatment.

\section{Declaration of interest}

M.C. participated as a member of the World Health Organization Working Group on the Classification of Disorders Specifically Associated with Stress, reporting to the International Advisory Group for the Revision of ICD-10 Mental and Behavioural
Disorders. However, the views expressed reflect the opinions of the author and not necessarily the Working Group or Advisory Group and the content of this article does not represent World Health Organization policy.

\section{Keywords}

Post-traumatic stress disorder; trauma; borderline personality disorder; individual psychotherapy; cognitive behavioural therapies.

\section{Copyright and usage}

(c) The Author 2020.
Marylène Cloitre (pictured) is the Associate Director of Research at the National Center for PTSD Dissemination and Training Division, VA Palo Alto Health Care System and a Clinical Professor (Affiliate) in the Department of Psychiatry and Behavioural Sciences at Stanford University. that the possible symptom combinations allow for 636120 ways to be diagnosed with DSM-5 PTSD, leading to potential complications in assessment and treatment planning.

\section{ICD definitions of PTSD}

\section{DSM definitions of PTSD}

The diagnosis of post-traumatic stress disorder (PTSD) was first introduced in 1980 in DSM-III. It differed from many DSM disorders in that it described symptoms that were the result of a traumatic event (for example combat, rape, vehicular accident) and required the presence of a traumatic stressor as a prerequisite for evaluation of the diagnosis. The first iteration of the PTSD diagnosis was comprised of three symptom clusters: re-experiencing of the trauma (for example nightmares, flashbacks), avoidance of reminders of trauma-related stimuli (for example places that reminded the person of the event, talking about the experience) and hypervigilance (for example easy startle, excessive attention to potential threat). The diagnosis proved highly influential leading to decades of research developing and testing treatments for PTSD, the most effective of which have been trauma-focused cognitive-behavioural therapies (CBTs), eye movement desensitisation and reprocessing and, to a lesser extent, pharmacological treatments, specifically selective serotonin reuptake inhibitors (SSRIs).

Despite the clinical benefits and popularity of the PTSD diagnosis, there have been persistent questions about whether its formulation in DSM is optimal. The symptom profile for the diagnosis in the current and fifth formulation of DSM (DSM-5) published in 2013, has grown to include 20 symptoms, 4 symptom clusters and a subtype for dissociation. The additions to the diagnosis have been an effort to recognise the heterogeneity of symptoms observed among various trauma populations. DSM-5 added a symptom cluster of negative alterations in mood and cognitions, expanded hypervigilance to include problems with anger and reckless behaviours and added dissociative experiences (derealisation, depersonalisation) as a subtype. One consequence of this expansion has been
In contrast, the eleventh revision of $\mathrm{ICD}^{1}$ (ICD-11), released in 2018 , has adopted a public health perspective that emphasises clinical utility including simplicity in diagnostic structure and transparent application to treatment planning. ${ }^{2}$ The symptom heterogeneity observed among trauma survivors has been recognised and organised into two disorders, PTSD and complex PTSD (CPTSD), ${ }^{3}$ with each disorder comprised of a relatively simplified set of symptom clusters and a conceptual organisation that appears easy for clinicians to follow. The two diagnoses are siblings under the parent category of 'Disorders specifically related to stress.' An individual can be diagnosed with either PTSD or CPTSD but not both; if a person is diagnosed with CPTSD, they cannot also have PTSD.

\section{Definition of CPTSD versus PTSD}

The ICD-11 formulation and characterisation of the two disorders follow from a long history of clinical observation that individuals who experienced chronic, repeated and prolonged traumas, such as childhood sexual abuse or domestic violence, tended to experience more complex reactions extending beyond those typically observed in PTSD and which included effects in three key domains: emotion regulation, self-identity and relational capacities. Thus, in ICD-11, CPTSD is a disorder that includes not only the symptoms of PTSD but additionally disturbances in the three above domains.

The ICD-11 formulation of PTSD requires exposure to a trauma defined as an extremely threatening or horrific event or series of events. Similar to the original DSM version of PTSD, the disorder includes three core elements or clusters: re-experiencing of the traumatic event in the present, avoidance of traumatic reminders and a sense of current threat. This formulation conceptualises PTSD primarily as a conditioned fear response and the re-experiencing and 
avoidance symptoms are specifically tied to the traumatic event. CPTSD includes the three core elements of PTSD as well as three additional elements called disturbances in self-organisation that are pervasive and occur across various contexts: emotion regulation difficulties (for example problems calming down), negative self-concept (for example beliefs about self as worthless or a failure) and relationship difficulties (for example avoidance of relationships).

Although the majority of investigations to date indicate that CPTSD typically emerges from sustained traumatic experiences, ${ }^{4}$ at least some have found that individuals with chronic traumas such as childhood abuse appear to develop PTSD and not CPTSD and conversely individuals with single incident traumas develop CPTSD. These observations led to consideration of the influence of dispositional (for example genetic make-up) or environmental (social support or lack thereof) factors in the development of the disorders such that trauma history is now recognised as a risk factor rather than a requirement for one or the other disorder. Vulnerable individuals who experience a single-incident trauma may develop CPTSD whereas a resilient or well-supported person with a history of sustained trauma might develop PTSD or neither disorder.

At this point, there is substantial evidence supporting the discriminative validity of PTSD and CPTSD diagnoses. In a 2017 review, ${ }^{4}$ the distinction between ICD-11 PTSD and CPTSD was supported in nine out of ten studies. Across epidemiological, community and clinical sample studies, symptom profile derived from latent class analyses clearly identified subgroups of the study populations following either a CPTSD profile or a PTSD profile and these profiles were distinguishable from one another. Since this review, several more studies have been published consistent with these results and include child and adolescent samples, refugee samples and Israeli prisoners of war. In addition, five studies evaluating functional impairment have reported that CPTSD was associated with more severe impairment than PTSD suggesting that the greater symptom load represented in CPTSD translated to greater difficulties in daily living. ${ }^{4}$

\section{CPTSD and borderline personality disorder}

There has been debate over nearly two decades as to whether CPTSD is actually PTSD with comorbid borderline personality disorder (BPD). Several studies using various statistical techniques have demonstrated that individuals with CPTSD are distinguishable from those with BPD. There are several notable clinical differences that have treatment implications. Individuals with CPTSD experience a severe but stable negative self-concept whereas those with $\mathrm{BPD}$ report shifts in their self-image vacillating between highly positive and highly negative self-perceptions. CPTSD relational difficulties are characterised by a tendency to avoid and have difficulty maintaining relationships, particularly during periods of conflict or high emotion whereas BPD is associated with rapid engagement followed by ups and downs or idealisation and devaluation of relationships. Although emotion regulation difficulties are central to both CPTSD and BPD, their expression can be quite different with suicide attempts and gestures and self-injurious behaviours a core feature and a first target of treatment for BPD in contrast to CPTSD, which does not include either problem for diagnosis and preliminary data indicate rates of these problems are substantially lower in CPTSD relative to $\mathrm{BPD}$.

\section{Prevalence of CPTSD and PTSD}

Research on the prevalence of CPTSD and PTSD in different countries, cultures and settings has just begun. This work has been substantially aided by the development of a validated self-report measure for ICD-11 PTSD and CPTSD, the International Trauma Questionnaire (ITQ) ${ }^{5}$ and a clinician interview, the International Trauma Interview for which reliability and validity data are accumulating. Factor analyses across different countries and cultures have consistently conformed with the conceptualisation of CPTSD as comprised of two symptom clusters, namely symptoms of PTSD and of disturbances in self-organisation. General population studies using the ITQ indicate that in high-income countries such as the USA, the UK, Germany and Israel the rates of PTSD and CPTSD combined range from 2.0 to $12.7 \%$, whereas in the low-income countries studied thus far in Africa (Ghana, Kenya and Nigeria), rates of the two disorders are higher ranging from 30.6 to $37.0 \%$.

\section{Interventions for CPTSD and PTSD}

The clinical utility of the presence of these two diagnoses has yet to be investigated. Given that CPTSD is comprised of a greater number and diversity of symptoms and associated with greater impairment, its treatment may require a greater number of interventions or a longer course of treatment. The development of different treatment plans relative to the complexity and severity of the diagnoses is consistent with a 'personalised medicine' approach and World Health Organization (WHO) guidelines include the twin goals of optimising outcome for the individual patient and deploying limited resources to those who need it most.

Given that CPTSD is a newly defined condition there are as yet no clinical trials evaluating interventions for its treatment. Studies exploring the relationship of CPTSD symptoms among each other in network analyses have identified negative self-concept as a central symptom, meaning that this symptom strongly influences and is influenced by other symptoms of the disorder. While we wait for evidence to emerge, it is appropriate to provide evidencebased PTSD treatments for both ICD-11 PTSD and CPTSD but also to consider additional interventions or therapies for CPTSD that deal with disturbances in self-organisation and include emotion regulation, cognitive re-appraisal and self-compassion.

Psychopharmacological interventions, particularly SSRIs and venlafaxine have been frequently used in the treatment of PTSD. However, recent meta-analyses have indicated that the effect sizes for SSRIs are low and inferior to those obtained for traumafocused CBTs. Given the current evidence, the use of medication is considered a second-line intervention to effective psychological treatments and, ideally, restricted to those medications with evidence of effect in PTSD. Reviews of the use of SSRIs for depression indicate that they are consistently less effective for individuals with childhood abuse, suggesting that their impact on PTSD or CPTSD in this population may similarly be limited. Nevertheless, the role of medications in combination with psychological therapies where synergistic or additive effects may emerge remain to be investigated.

\section{Next steps}

Continued exploration of the risk factors that differentiate PTSD and CPTSD as well as the discriminant validity of CPTSD relative to other psychiatric disorders is valuable in order to better characterise and further validate the disorders. Important next steps include conducting ICD-11 and DSM diagnoses with reliable measures in the same populations to determine the overlap between those diagnosed with ICD-11 PTSD and CPTSD as compared with DSM. It is expected that the overlap will be large, but that the ICD-11 will provide a more refined characterisation of patient 
populations via the differential diagnoses of PTSD and CPTSD. Both DSM and ICD diagnoses are recognised by third party payors for mental health services, so either diagnostic approach can be use. The potential benefit in using the ICD system is expected to be in its translation to treatment protocols that are tailored to the symptom profiles and level of impairment associated with each disorder. The development and testing of such protocols is a critical next step in the ICD-11 research agenda.

Although investigations thus far indicate consistency in the presence of a PTSD/CPTSD distinction across several countries, evaluation across other regions of the globe is important as is assessment of invariance in the currently accepted symptom profiles for each disorder. Refinement of the organisation and nature of the symptoms associated with each disorder may need to be considered as more research is conducted.

Marylène Cloitre (D), National Center for PTSD Dissemination and Training Division, VA Palo Alto Health Care System, USA; and Department of Psychiatry and Behavioural Sciences, Stanford University, USA

Correspondence: Marylène Cloitre. Email: marylene.cloitre@va.gov

First received 6 Dec 2019, final revision 15 Jan 2020, accepted 22 Jan 2020

\section{Supplementary material}

Supplementary material providing additional citations related to the above text is available online at https://doi.org/10.1192/bjp.2020.43

\section{References}

1 World Health Organization. International Statistical Classification of Diseases and Related Health Problems (11th Revision). WHO, 2018.

2 Reed GM. Toward ICD-11: improving the clinical utility of WHO's international classification of mental disorders. Prof Psychol Res Pract 2010; 41: 457.

3 Maercker A, Brewin CR, Bryant RA, Cloitre M, Reed GM, van Ommeren M, et al. Proposals for mental disorders specifically associated with stress in the International Classification of Diseases-11. Lancet 2013; 381: 1683-5.

4 Brewin CR, Cloitre M, Hyland P, Shevlin M, Maercker A, Bryant RA, et al. A review of current evidence regarding the ICD-11 proposals for diagnosing PTSD and complex PTSD. Clin. PSychol. Rev 2017; 58: 1-5.

5 Cloitre M, Shevlin M, Brewin CR, Bisson Jl, Roberts NP, Maercker A, et al. The International Trauma Questionnaire: development of a self-report measure of ICD-11 PTSD and complex PTSD. Acta Psychiatr Scand 2018; 138: 536-46.

\section{psychiatry in history}

\section{The Amsterdam Suggestive Psychotherapy Clinic}

\section{Stephen Wilson (iD)}

In the 6 years between 1887 and 1893, Drs A. W. Van Renterghem and F. Van Eeden treated more than a thousand patients. They published their results in French in Psycho-Therapie (1894). ${ }^{1}$ Its larger purpose was to counter prevailing materialistic conceptions of the human mind. Both authors allied themselves to the therapeutic methods of Ambroise August Liébeault and Hyppolite Bernheim. But they wanted to distance themselves from the dramatic hypnotic séances given by Jean-Martin Charcot in Paris, and the dubious, but undeniably popular, activities of various stage hypnotists, magnetists and mountebanks. For this reason they preferred the use of the term psychotherapy.

Several years ago $^{2}$ we maintained that the first concern of the doctor practicing psychotherapy should be to try to increase the psychological stability of his patient. We had no argument with the view that a certain link exists between hysteria and hypnosis or suggestibility, and we thought it could be detected in the psychological dissociation or instability which is innate in the hysteric and artificially induced in the hypnotised.

But at the same time we pointed to the deplorable fact that psychotherapy, whose theoretical basis was founded by Hack Tuke and whose practical application we learned from Liébault, has been confused and conflated with hypnotism. In fact, the two should be distinguished as clearly as possible. We are absolutely convinced that the word hypnotism, in Charcot's sense, has nothing to do with psychotherapy, and only produces confusion.

The only clear and durable way of conceptualising psychotherapy seems to us to be the following: that psychotherapy combats illness through the mobilisation of the patient's psychological organ; suggestion, exercise and strengthening through encouragement are its instruments. This conception would surely be seen as an obvious fact, and the most innocent in the world, were it not for the confusion brought about by the abnormal practices of hypnotism.

That it is possible to induce a state of sleep in many patients by verbal influence, and that experience teaches us the influence exercised over a sleeping person or one half asleep, or in a passive state, acts more powerfully than in the waking state; constitutes moreover a simple fact, not in any way frightening, and is a useful ingredient in the application of psychotherapy. It permits us to apply psychological therapy methodically.

For the doctor who practises psychotherapy, hypnotic experiments simply constitute a warning that he could harm his patients if he doesn't stick strictly to therapeutic aims. That is to say, he could increase abnormal suggestibility and thus produce an undesirable lasting state of instability, whereas normal suggestibility is absolutely sufficient for his ends, notably cure.

\section{References}

1 Société d'èditions scientifiques, 1894. In Wilson S. (trans.) Psycho-Therapy. Lamad Vav Press, 2019.

2 van Eeden F. The Principles of Psycho-Therapy. London International Congress of Experimental Psychology, 1892. 